\title{
Single electron-phonon interaction in a suspended quantum dot phonon cavity
}

\author{
E. M. Höhberger ${ }^{1}$ R. H. Blick ${ }^{1}$ 母 T. Brandes ${ }^{2}$, J. Kirschbaum ${ }^{1}$, W. Wegscheider ${ }^{3}$, M. Bichler ${ }^{4}$, and J. P. Kotthaus ${ }^{1}$ \\ ${ }^{1}$ Center for NanoScience \& Sektion Physik, Ludwig-Maximilians-Universität, 80539 München, Germany \\ 2 Department of Physics, University of Manchester, \\ Institute of Science and Technology (UMIST), Manchester M60 1QD,UK \\ 3 Institut für Angewandte und Experimentelle Physik, \\ Universität Regensburg, 93040 Regensburg, Germany and \\ 4 Walter-Schottky-Institut, Technische Universität München, 85748 Garching, Germany
}

(Dated: October 8, 2019)

\begin{abstract}
An electron-phonon cavity consisting of a quantum dot embedded in a free-standing GaAs/AlGaAs membrane is characterized in Coulomb blockade measurements at low temperatures. We find a complete suppression of single electron tunneling around zero bias leading to the formation of an energy gap in the transport spectrum. The observed effect is induced by the excitation of a localized phonon mode confined in the cavity. This phonon blockade of transport is lifted at magnetic fields where higher electronic states with nonzero angular momentum are brought into resonance with the phonon energy.

PACS numbers: 73.23.Hk, 73.63.Kv, 73.21.La, 71.38.-k, 62.25.+g, 85.85.+j
\end{abstract}

When Rolf Landauer discussed the importance of irreversibility and heat generation for classical transistors in 1961 1], he found that the minimal amount of dissipation required to perform a single bit operation is given by $k_{\mathrm{B}} T \ln 2$. In the context of quantum physics the role of dissipation needs to be reconsidered. Even more, along with the discovery of single electron transistors [2, 3] and quantum dots [4, 5], it is nowadays regarded as crucial for the feasibility of quantum computation [6, 7]. The next step towards control of dephasing of electronic quantum states is tailoring phonon confinement in quantum dot cavities. The most promising approach to address the physics of dissipation in the ultimate limit of single electrons interacting with individual phonon modes of their host crystal is to embed a low-dimensional electron gas into a suspended phonon cavity as demonstrated in previous work $[8,9,10]$. The relevance of the electronphonon interaction for quantum dot systems was initially explored by Fujisawa et al. 11] and Qin et al. 12 and theoretically confirmed by Brandes and Kramer [13].

Already back in 1967, Duke et al. 14] modelled inelastic tunneling through a barrier, finding that collective phonon modes can be excited by the tunneling electron. According to their calculations characteristic zero bias conductance minima in the tunnel conductance can be attributed to this effect. In recent years, electron back-action on mechanical degrees of freedom has been theoretically discussed by Schwabe et al. 15 and Blencowe [16. The additional implications of phonon confinement in an electron-phonon cavity were modelled by Debald et al. 17], pointing out the possibility to control electron dephasing by tailoring the phonon spectrum.

Here we report on the experimental observation of a new blocking mechanism of single electron transport which is found in such a cavity, evidencing the coherent interplay between single electron tunneling and the excitation of localized phonon modes confined in the cavity as predicted by Duke et al. 14]. Strikingly similar features which can be attributed to the same effect have been observed for single electrons tunneling onto a $\mathrm{C}_{60}$ molecule by Park et al. [18, 19]. The underlying physics of coherently coupling discrete electronic states with discrete phonon modes bears resemblance to cavity QED [20].

A phonon cavity containing a suspended single quantum dot is shown in Fig. 1(a). The scanning electron micrograph was taken under an angle of $65^{\circ}$ in order to visualize the three-dimensional character of the sample. Depicted in blue is the free-standing $130 \mathrm{~nm}$ thick GaAs/AlGaAs membrane containing a confined electron gas which is located $40 \mathrm{~nm}$ below the sample surface. The $400 \mathrm{~nm}$ thick sacrificial layer of $\mathrm{Al}_{0.8} \mathrm{Ga}_{0.2}$ As supporting the membrane has been completely removed beneath the displayed part of the sample in Fig. 1(a) creating a spacing between the membrane and the GaAs buffer displayed in grey. The quantum dot is defined in a $600 \mathrm{~nm}$ wide bar by two point contacts formed by pairs of symmetric indentations. As a result of edge depletion the cavity has a reduced electronic diameter of about $450 \mathrm{~nm}$ [10]. The two constrictions are wide enough to allow ballistic transport through the cavity, but can be depleted to form tunneling barriers by a nearby gate electrode. In the case of Fig. 1(a) this task is accomplished by a close-by Hallbar being employed as an in-plane gate. A schematic top-view of the sample layout is displayed in the inset.

The presented measurements are performed in a dilution refrigerator with a base temperature of $T_{\text {bath }}=$ $10 \mathrm{mK}$. A negative voltage $V_{\mathrm{g}}$ is applied to the gate electrode (i.e. the Hall-bar in Fig. 1(a)) in order to create tunneling barriers and to vary the electrochemical potential of the dot denoted $\mu(N+1)=E(N+1)-E(N)$ in the level diagrams of Fig. 1(b) which will be discussed in more detail below. A bias voltage $V_{\text {sd }}$ can be applied between the source and drain reservoirs. The differential conductance $G=d I_{\mathrm{sd}} / d V_{\text {sd }}$ is recorded with respect 


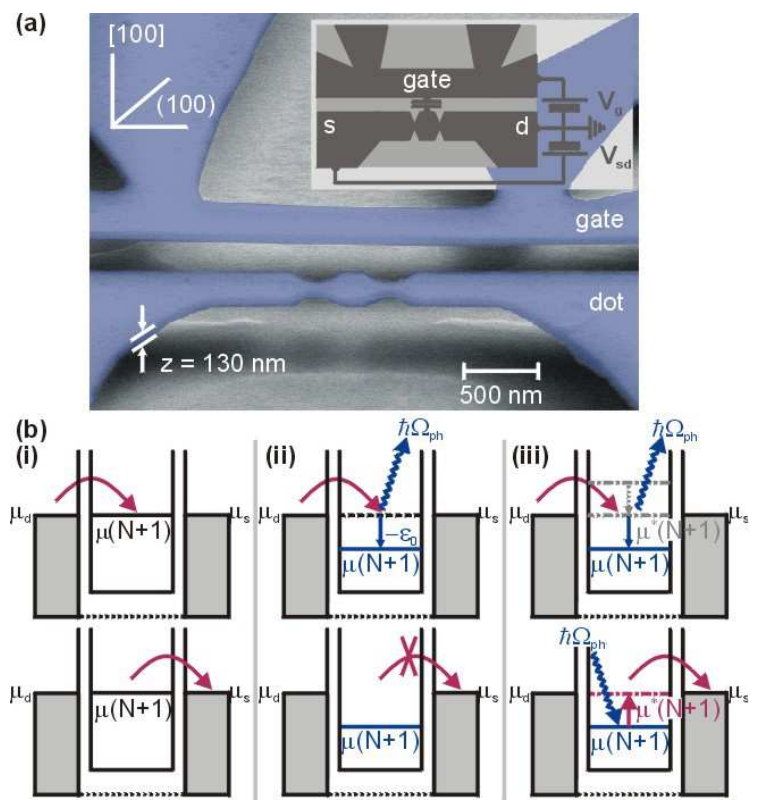

FIG. 1: (a) Suspended quantum dot cavity and Hall-bar formed in the blue-colored $130 \mathrm{~nm}$ thin GaAs/AlGaAs membrane. The inset shows a schematic top view of the sample. (b) Level diagrams for single electron tunneling including phonon blockade: (i) In the orthodox model electrons sequentially tunnel through the dot, if the chemical potential $\mu(N+1)$ is aligned between the reservoirs. (ii) Tunneling into the phonon cavity results in the excitation of a cavity phonon with energy $\hbar \Omega_{\mathrm{ph}}$, leading to a level mismatch $\epsilon_{0}$ and thus to phonon blockade. (iii) Single electron tunneling is reestablished by a higher lying electronic state $\mu^{*}(N+1)$ which is enabled to coherently reabsorb the phonon and to hereby replace the ground state [28].

to both $V_{\mathrm{g}}$ and $V_{\mathrm{sd}}$ showing clear Coulomb diamonds $[5$ depicted in logarithmic color scale representation in the left part of Fig. 22 (blue: $0.02 \mu \mathrm{S}$, red: $6 \mu \mathrm{S}$ ). The right part of the figure displays the corresponding line plots at zero bias $V_{\mathrm{sd}}=0 \mu \mathrm{V}$. Figure 2(a) was taken at an electron temperature of $T_{\mathrm{e}}=100 \mathrm{mK}$ and a perpendicular magnetic field of $B=500 \mathrm{mT}$ where a quasi-continuum of energetically higher states produces a Coulomb blockade diamond known from conventional quantum dots $[5]$. The charging energy $E_{\mathrm{C}}=e^{2} / 2 C_{\Sigma}=0.56 \mathrm{meV}$ corresponds to a dot capacitance of $C_{\Sigma}=140 \mathrm{aF}$ from which a dot radius of $r=160 \mathrm{~nm}$ and an electron number of about 480 can be deduced. The striking difference as compared to conventional quantum dot measurements is observed for the same temperature but zero magnetic field where we find complete suppression of conductance around zero bias 21]. This is shown exemplarily for three adjacent resonances in Fig. 2(b), both from the gap opening between the diamonds in the color scale plot and from the lineplot showing zero conductance 22]. The resulting blockade of transport can only be overcome by applying a positive or negative source drain bias of $\left|V_{\mathrm{sd}}\right|=100 \mu \mathrm{V}$ (see also Fig. 3(a) magnifying a single resonance).
These results can be compared to recent theoretical models for transport through a molecular single electron transistor coupled to a single vibrational mode 23, 24, 25. We have extended these models 26 in order to analyze the origin of the observed blockade mechanism. The picture corresponding to the classical limit (strongly overdamped vibration mode) is illustrated in Fig. 1(b): (i) In case of a conventional single electron transistor an electron sequentially tunnels through a non-suspended quantum dot whenever Coulomb blockade can be overcome, i.e. the electrochemical potentials of source, drain, and the $\operatorname{dot} \mu_{s}, \mu_{d}$ and $\mu(N+1)$ are aligned. This situation corresponds to the well-known Mössbauer-effect [27] in gamma spectroscopy: Recoil-free absorption and emission of gamma-ray photons is enabled for a nucleus being placed in a solid since the crystal takes up the recoil as a whole without entailing an energy loss. In a classical picture this is equivalent to the reflection of a particle at a hard wall with infinite mass. (ii) This behavior changes dramatically for a quantum dot embedded in a suspended phonon cavity a classical analogue of which is given by a particle hitting a trampoline. Due to strong electronphonon coupling in the cavity [17] (see below), single electron tunneling induces mechanical displacement of the suspended quantum dot which corresponds to the excitation of a localized cavity phonon of energy $\hbar \Omega_{\mathrm{ph}}$. Flensberg has shown 25] that in the strongly overdamped, classical limit (quality factor $Q=\Omega_{\mathrm{ph}} / \gamma \ll 1$ for small phonon life time $\gamma^{-1}$ ), the energy cost of the displacement goes along with a drop of the chemical potential $\mu(N+1)$ in the dot, which leads to a blockade of single electron tunneling. The energy gap $\epsilon_{0}=g \hbar \Omega_{\mathrm{ph}}$ then depends on the Franck-Condon coupling constant $g$. In the 'Mössbauer picture' 14], the cavity picks up the 'recoil' of the tunneling electron and immediately relaxes to a new ground state for $Q \rightarrow 0$. On the other hand, $Q \rightarrow \infty$ would correspond to coherent cavity phonons where the Franck-Condon factors yield a series of phonon side-bands $n \Omega$ with weights given by the Poisson distribution $e^{-\mathrm{g}} g^{\mathrm{n}} / n$ ! at zero temperature with $n=0$ corresponding to elastic tunneling.

To re-establish single electron tunneling, the energy transferred to the cavity can be regained as displayed in part (iii) of Fig. प(b) where the cavity phonon is reabsorbed exciting a higher lying electronic state $\mu^{*}(N+1)[28]$. To this end, the excited cavity phonon mode coherently exchanges energy with the electronic excited states $\mu^{*}(N+1)$ which can be understood in terms of (damped) Rabi oscillations [26].

An estimate for the phonon modes and energies confined to the quantum dot cavity is obtained from microscopic calculations 17 that find van-Hove singularities in the cavity phonon density of states, accompanied by an extreme enhancement of phonon emission at certain phonon energies. The lowest energy where this occurs is $\hbar \Omega_{\mathrm{ph}} \approx 3 \hbar c_{\mathrm{L}} / z=73 \mu \mathrm{eV}$ for quantized dilatational 


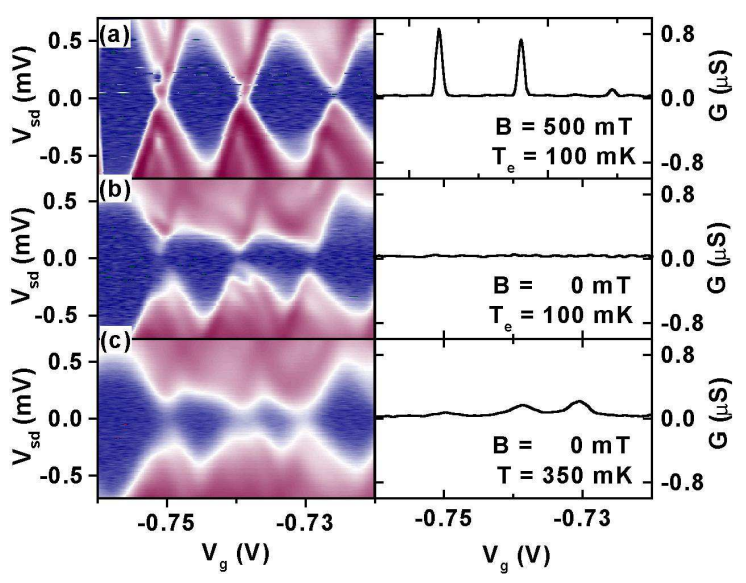

FIG. 2: Transport spectrum of the suspended single quantum dot and zero bias conductance: (a) Single electron resonances taken at an electron temperature of $100 \mathrm{mK}$ and a perpendicular magnetic field of $500 \mathrm{mT}$. (b) At zero magnetic field conductance is suppressed for bias voltages below $100 \mu \mathrm{V}$ due to phonon excitation. (c) The conductance pattern at $350 \mathrm{mK}$ shows that phonon blockade starts to be surpassed because of thermal broadening of the Fermi function supplying empty states in the reservoirs.

phonon modes ( $\hbar \Omega_{\mathrm{ph}} \approx 145 \mu \mathrm{eV}$ for flexural modes) for the sample thickness $z=130 \mathrm{~nm}$ and the longitudinal velocity of sound in the [100] direction of bulk GaAs, $c_{\mathrm{L}}=4.77 \cdot 10^{5} \mathrm{~cm} / \mathrm{s}$. This prediction for $\hbar \Omega_{\mathrm{ph}}$, based on a simple infinite thin-plate model, compares relatively well to the observed energy gap $\epsilon=100 \mu \mathrm{eV}$ found in our transport data at an electron temperature of $100 \mathrm{mK}$. At larger temperatures the electrons gain enough energy to overcome phonon blockade when the broadening of the Fermi distribution function in the leads approaches $4 k_{\mathrm{B}} T \approx \epsilon_{0}$. This is observed in Fig. 2( c), where clear single electron tunneling resonances are found again at $T=350 \mathrm{mK}$ and $B=0 \mathrm{~T}$. We therefore conclude that quantized cavity phonons are excited within the cavity.

The phonon gap $\epsilon$ is recorded with a higher resolution in Fig. 3(a) showing the central region of Fig. 2(a) in the same color scale. The conventional shape of the Coulomb diamonds is marked by solid lines. Clearly, the asymmetric shape of the gap can be discerned: The crossing of the two solid lines indicates the position of the missing single electron tunneling conductance peak. The onset of conductance $(*)$ occurs at a slightly different value of $V_{\mathrm{g}}$ by which the half diamonds are offset as indicated by dotted lines. This offset is also explained by the model developed in Fig. 廿(b): At the onset of conductance at $V_{\mathrm{sd}}=\epsilon / e$ the energy level $\mu(N+1)$ only remains in the transport window after the level mismatch when initially being aligned with the upper reservoir. Hence, the initial level position is shifted by $\delta V_{\mathrm{g}}=\epsilon / 2 e \alpha$ (where $\left.\alpha=C_{\mathrm{g}} / C_{\Sigma}\right)$ on the gate voltage axis.

Further evidence for phonon blockade is given by tracing the magnetic field dependence of the conduc-

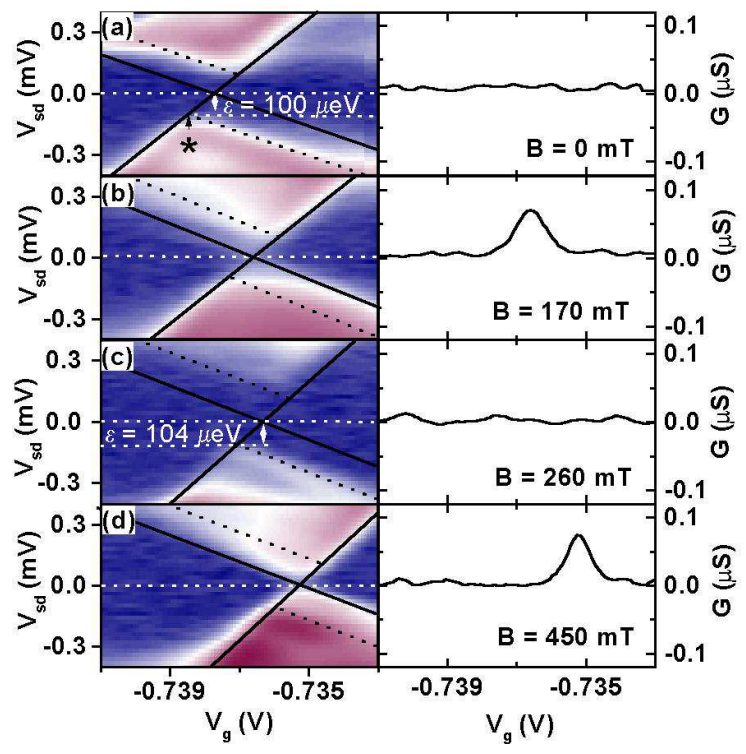

FIG. 3: Transport spectrum for (a) $B=0 \mathrm{mT}$, (b) $170 \mathrm{mT}$, (c) $260 \mathrm{mT}$, and (d) $450 \mathrm{mT}$. The line plots give the zero bias trace. At certain magnetic fields (b,d) excited quantum dot states with higher magnetic momentum are brought into resonance with the cavity phonon re-enabling single electron tunneling. Otherwise $(\mathrm{a}, \mathrm{c})$ transport is suppressed due to phonon blockade with an excitation barrier of around $100 \mu \mathrm{eV}$.

tance gap. Energetically higher lying electronic states $\mu^{*}(N+1)$ with finite angular momentum $\ell \cdot \hbar(\ell=1,2, \ldots)$ can be brought into resonance with the cavity phonon by altering the magnetic field such that the excitation energy $\mu^{*}(N+1, B)-\mu(N+1, B=0)=\epsilon_{0}$, corresponding to the situation displayed in level diagram (iii) of Fig. 1(b). In this case, the higher states offer a broad cross section for the emitted phonons to be reabsorbed, allowing for the excitation of the electron into $\mu^{*}(N+1)$ thus enabling it to sustain single electron tunneling. Examples for such resonances can be seen at $170 \mathrm{mT}$ and $450 \mathrm{mT}$ shown in Fig. 3(b) and (d) whereas a non-resonant situation at $260 \mathrm{mT}$ is displayed in Fig. 31 (c). Since reabsorption of the phonon only occurs with a finite probability, the height of the conductance peak at zero bias, which is again displayed in the right part of the figure, is reduced compared to the onset of conductance through the original ground state at $V_{\mathrm{sd}}=\epsilon / e$ marked by the dotted line as described before.

A direct comparison of the magnetic field dependence to transport spectroscopy on the dot is depicted in Fig. [4 where we consider two adjacent resonance peaks $\alpha$ (right, c.f. Fig. 3) and $\beta$ (left). The conductance traces are recorded for bias voltages from $0 \mu \mathrm{V}$ to $-800 \mu \mathrm{V}$ at $B=0 \mathrm{mT}$ in Fig. 四(a) showing excited states marked by red lines. The zero bias conductance is plotted logarithmically as a function of both gate voltage $V_{\mathrm{g}}$ and magnetic field $B$ for $\alpha$ and $\beta$ in Fig. 四(b) (blue: $0.02 \mu \mathrm{S}$, red: $0.2 \mu \mathrm{S}$ ) and (c) (blue: $0.02 \mu \mathrm{S}$, red: $2 \mu \mathrm{S}$ ), respectively. For the right resonance $\alpha$ we find excited states 


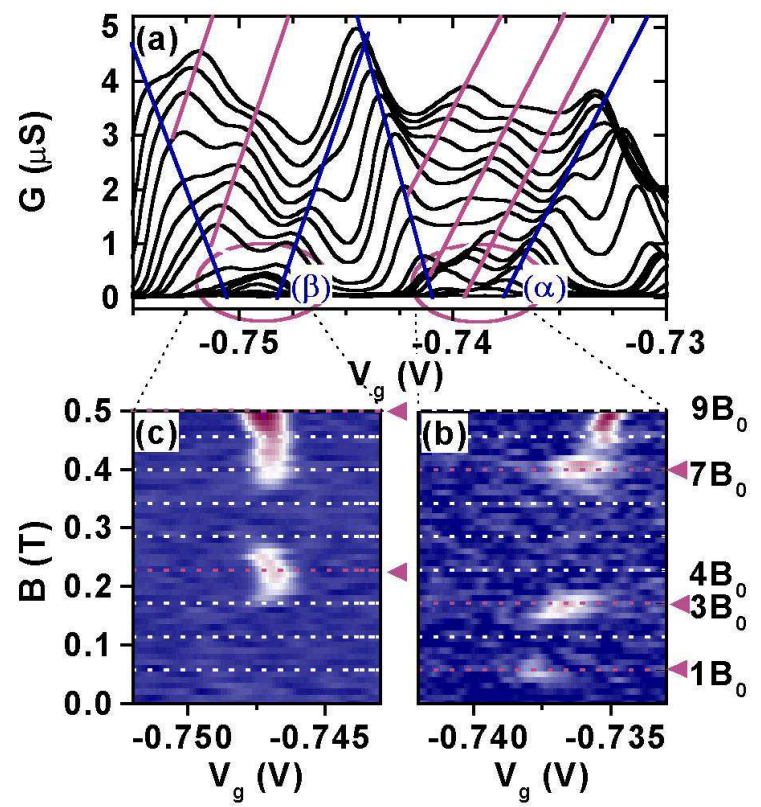

FIG. 4: (a) Line plot of conductance resonances $\alpha$ and $\beta$ at different source-drain bias voltages between $0 \mu \mathrm{V}$ and $-800 \mu \mathrm{V}$. Blue lines follow the ground states, while red lines mark excited states. The conductance at $V_{\mathrm{sd}}=0 \mu \mathrm{V}$ is suppressed. (b) Zero bias conductance for resonance $\alpha$ plotted against gate voltage $V_{\mathrm{g}}$ and magnetic field $B$. Finite conductance appears for $57 \mathrm{mT}, 170 \mathrm{mT}$, and $400 \mathrm{mT}$. (c) Similar plot for resonance $\beta$ (blue: $0.02 \mu \mathrm{S}$, red: $2 \mu \mathrm{S}$ ): Non-zero conductance is found for $230 \mathrm{mT}$ and $510 \mathrm{mT}$.

(red lines) at energies $\mu_{\alpha}^{* 1}=230 \mu \mathrm{eV}, \mu_{\alpha}^{* 2}=440 \mu \mathrm{eV}$, and $\mu_{\alpha}^{* 3}=740 \mu \mathrm{eV}$. These three excited states correspond to three magnetic fields permitting zero bias conductance at $57 \mathrm{mT}, 170 \mathrm{mT}$, and $400 \mathrm{mT}$. For the left resonance $\beta$ we find excited states at energies $\mu_{\beta}^{* 1}=380 \mu \mathrm{eV}$ and $\mu_{\beta}^{* 2}=760 \mu \mathrm{eV}$ matching with two magnetic fields reenabling zero bias conductance at $230 \mathrm{mT}$ and $510 \mathrm{mT}$. Above $500 \mathrm{mT}$ conductance fully re-emerges while below it is found only at a set of discrete values as discussed above. These values exactly correspond to multiples $B=n \cdot B_{0}(n=1,3,4,7$ and 9$)$ of the first resonance in $\alpha$ observed at $B_{0}=57 \mathrm{mT}$.

The presented measurements demonstrate that for freely suspended quantum dot cavities single electron tunneling gives rise to the excitation of a longitudinal cavity phonon. The resulting energy loss leads to a suppression of linear electron transport and to the formation of a distinct energy gap. This phonon blockade effect can be overcome at bias voltages large enough to bridge the energy gap, or at a sufficiently high bath temperature. A third mechanism circumventing phonon blockade is given by aligning higher lying electronic states with distinct angular momentum such that electronic transport is enabled through these states after reabsorption of the cavity phonon in a process similar to Rabi oscillations. Finally, placing the dot into the whole bulk crystal in- stead of a free-standing cavity eliminates the blockade effect so that elastic single electron tunneling is restored.

We like to thank Stefan Debald for stimulating discussions. Support from the Bundesministerium für Forschung und Technologie and the Deutsche Forschungsgemeinschaft is acknowledged.

* Electronic address: hoehberger@lmu.de

$\dagger$ New present address: Electrical and Computer Engineering, University of Wisconsin-Madison, Madison, Wisconsin 53706, USA.

[1] R. Landauer, IBM J. Res. Develop. 5, 183 (1961).

[2] T. A. Fulton and G. J. Dolan, Phys. Rev. Lett. 59, 109 (1987).

[3] H. Grabert and M. H. Devoret, Single Charge Tunneling (Plenum, New York, 1992).

[4] R. Ashoori, Nature 379, 413 (1996).

[5] L. P. Kouwenhoven et al., Mesoscopic Electron Transport (Kluwer Academic, Dordrecht, 1997), p. 105-214.

[6] D. V. Averin, Nature 398, 748 (1999).

[7] C. H. Bennett and D. P. DiVincenzo, Nature 404, 247 (2000).

[8] R. H. Blick et al., Phys. Rev. B 62, 17103 (2000).

[9] E. M. Höhberger et al., Physica E 12, 487 (2002).

[10] J. Kirschbaum et al., Appl. Phys. Lett. 81, 280 (2002).

[11] T. Fujisawa et al., Science 282, 932 (1998).

[12] H. Qin et al., Phys. Rev. B 64, R241302 (2001).

[13] T. Brandes and B. Kramer, Phys. Rev. Lett. 83, 3021 (1999).

[14] C. B. Duke, S. D. Silverstein, and A. J. Bennett, Phys. Rev. Lett. 19, 315 (1967).

[15] N. F. Schwabe et al., Phys. Rev. B 52, 12911 (1995).

[16] M. P. Blencowe, Physica B 263-264, 459 (1999).

[17] S. Debald, T. Brandes, and B. Kramer, Phys. Rev. B 66, R041301 (2002).

[18] H. Park et al., Nature 407, 57 (2000).

[19] P. McEuen, private communication.

[20] A. Imamoglu et al., Phys. Rev. Lett. 83, 4204 (1999).

[21] This is clearly different from spin-blockade (see A. K. Huettel et al., submitted to Europhys. Lett. (2002); cond-mat/0109014) where suppression of conductance only occurs at a single resonance peak due to the internal electron spin structure of a quantum dot.

[22] The curvilinear diamond shape in the high bias regime of Fig. 2 (b) is not related to phonon blockade. Distorted Coulomb diamonds have been discussed by Tans et al., Nature 394, 761 (1998) or Sapmaz et al., cond-mat/0206311

[23] D. Boese and H. Schoeller, Europhys. Lett. 54, 668 (2001).

[24] J.-X. Zhu and A. V. Balatsky, cond-mat/0210003

[25] K. Flensberg, cond-mat/0302193 cond-mat/0303236

[26] T. Brandes et al., in preparation.

[27] R. L. Mössbauer, Rev. Mod. Phys. 36, 362 (1964).

[28] In our case, higher lying electronic states $\mu^{*}(N+1)$ with non-zero angular momentum $\ell \cdot \hbar(\ell=1,2, \ldots)$ are considered the excitation energy of which can be brought into resonance with the cavity phonon by means of an applied perpendicular magnetic field. 